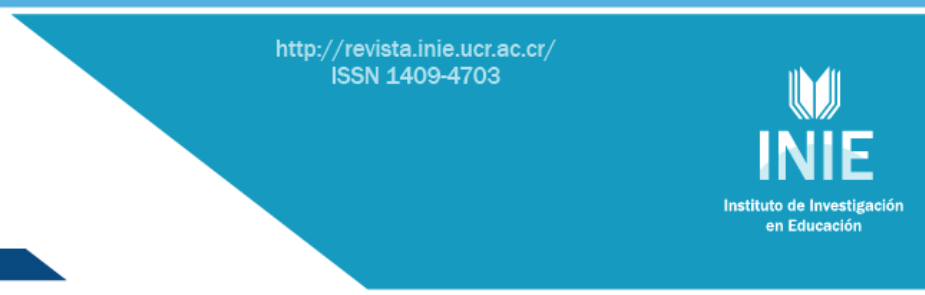

\title{
RECURSOS COMUNICATIVOS DE UN JOVEN CON AUTISMO: ENFOQUE MULTIMODAL PARA MEJORAR LA CALIDAD DE VIDA \\ COMMUNICATIVE RESOURCES OF A YOUNG PERSON WITH AUTISM: \\ MULTIMODAL APPROACH FOR A BETTER QUALITY OF LIFE
}

\section{Volumen 15, Número 1}

Enero - Abril

pp. 1-23

DOI: $\underline{\mathrm{dx} . \text { doi.org/10.15517/aie.v15i1.17592 }}$

Gerardo Godoy Echiburú

Dominique Manghi Haquin

Gonzalo Soto Cardenas

Isabel Aranda Godoy

Revista indizada en REDALYC, $\underline{\text { SCIELO }}$

Revista distribuida en las bases de datos:

CATÁLOGO DE LATINDEX, IRESIE, CLASE, DIALNET, DOAJ, E-REVIST@S, SHERPA/ROMEO, QUALIS, MIAR

Revista registrada en los directorios:

ULRICH'S, REDIE, RINACE, OEI, MAESTROTECA, PREAL, CLACSO 


\title{
RECURSOS COMUNICATIVOS DE UN JOVEN CON AUTISMO: ENFOQUE MULTIMODAL PARA MEJORAR LA CALIDAD DE VIDA COMMUNICATIVE RESOURCES OF A YOUNG PERSON WITH AUTISM: MULTIMODAL APPROACH FOR A BETTER QUALITY OF LIFE
}

\author{
Gerardo Godoy Echiburú ${ }^{1}$ \\ Dominique Manghi Haquin ${ }^{2}$ \\ Gonzalo Soto Cardenas ${ }^{3}$ \\ Isabel Aranda Godoy ${ }^{4}$
}

\begin{abstract}
Resumen: En el ámbito pedagógico, los aportes de la Semiótica Social, la Lingüística Sistémico Funcional y la Multimodalidad se presentan como herramientas teórico-metodológicas para analizar las interacciones comunicativas en contexto escolar. Dentro de la diversidad de experiencias y modos de comunicación que se presentan en la escuela existen unos muy particulares, el de las personas del espectro autista. Este estudio aborda la comunicación en la escuela desde el paradigma de la educación inclusiva y tiene como objetivo describir los recursos semióticos utilizados por un joven del espectro autista de una escuela especial de la comuna de Viña del Mar, Chile. Para esto se realiza un estudio de caso en el cual se registra de manera audiovisual la interacción escolar en un momento del día, durante cuatro semanas continuas. Para el análisis multimodal, se consideran las nociones de campo, tenor y modo junto con el intercambio de significados (metafunción interpersonal), enfocándose principalmente en el recurso "tirarse al suelo", siendo éste el más frecuente y cuyas funciones se presentan mayormente diferenciadas. Se identifican los recursos empleados para las dos opciones básicas del intercambio: oferta y/o demanda, ya sea de bienes y servicios o información, en los cuales el joven hace un uso consistente en diversos contextos escolares. Entre las proyecciones destaca que, a partir de la interpretación de sus interacciones, se puede mejorar la Calidad de Vida del estudiante propiciando un clima de aula favorable para el aprendizaje, validando sus interacciones no como conductas disruptivas si no como recursos comunicativos reconocidos por su entorno inmediato.
\end{abstract}

Palabras clave: AUTISMO, INCLUSIÓN, MULTIMODALIDAD, SEMIÓTICA SOCIAL, CHILE

\begin{abstract}
In the teaching area, the contributions done by Social Semiotics, Systemic Functional Linguistics and Multimodality are presented as theoretical and methodological tools to analyze the communicative interactions in the teaching context. Among the diversity of experiences and ways of communication that are presented in schools, the ones related to autism can also be found. This study approaches school from the inclusive education paradigm, it's main objective is to describe the semiotic resources being used by a student with autism spectrum attending to a special education school in Viña del Mar, Chile. This case study research is carried out by videotaping the social interaction of the student during four weeks in a row. For the multimodal analysis, the notions of field, tenor and mode are being considered along with the exchange of meaning (interpersonal metafunction), mainly focusing in the resource "down to the floor", being the latter the most frequent one and which functions are strongly differentiated. The main resources being identified answer to the two basic options of exchange: offer and/or demand, either goods and services or information, which the student makes constant use of among the different school settings. Encompassed by the projections, it can be highlighted by the different interpretations of interaction, that the student's quality of life can indeed be enhanced, providing a favorable learning setting; thus, validating the student's interactions not as a disruptive behavior but as a primary resource of communication known by their immediate environment.
\end{abstract}

Key words: AUTISM, INCLUSION, MULTIMODALITY, SOCIAL SEMIOTICS, CHILEAN EDUCATION, CHILE

\footnotetext{
Profesor en Escuela Especial Juanita Aguirre, Chile. Dirección electrónica: ichigen@gmail.com

2 Académica en Pontificia Universidad Católica de Valparaíso. Chile e Investigadora responsable del proyecto fondecyt1130684, Chile. Dirección electrónica: dmanghi@gmail.com

${ }^{3}$ Egresado de la carrera de Educación Diferencial, Pontificia Universidad Católica de Valparaíso.Chile. Dirección electrónica: gonzalo.soto.cardenas@gmail.com

${ }^{4}$ Estudiante de la carrera de Educación Diferencial en la Pontificia Universidad Católica de Valparaíso. Chile. Dirección electrónica: isabel.arandag@gmail.com
}

Artículo recibido: 7 de marzo, 2014

Enviado a corrección: 12 de setiembre, 2014

Aprobado: $1^{\circ}$ de diciembre, 2014 


\section{Introducción}

En la actualidad, el paradigma inclusivo propone construir una sociedad que valore la diversidad, poniendo énfasis en la modificación del entorno para facilitar la inclusión de las personas con necesidades de apoyo (Booth y Ainscow, 2002). Uno de los grupos que conforma la diversidad humana son las personas con discapacidad y dentro de ellas, las personas con autismo..La interacción de la persona que posee rasgos del espectro autista con su entorno social genera intercambios de significados muy particulares (Riviere, 2000), produciendo situaciones comunicativas en las cuales los significados son interpretados por ambos interlocutores de manera diferente (Halle, Brady y Drasgow, 2004).

Esta diferencia puede producir dos tipos de consecuencias. Por una parte, la persona con autismo sostiene en el tiempo su conducta comunicativa-verbal o no verbal hasta que su interlocutor logra interpretar su intención y contenido (Dreyfus, 2006). Por otra parte, ante esta conducta peculiar y persistente el interlocutor puede generar la creencia de que la persona con espectro autista no se está comunicando o que lo hace de forma inadecuada, a partir de los propios juicios sobre la manera apropiada en que las personas se deben comunicar (Quirós, 2006).

Dado lo anterior, este estudio se centra en la interacción de un estudiante del espectro autista en situación escolar, poniendo el foco en el repertorio de recursos que utiliza para comunicarse. En esta investigación se deben tomar en cuenta dos factores. El primer factor tiene que ver con la génesis del estudio: este trabajo surge desde las necesidades comunicativas en el aula de una escuela de Educación Especial chilena que atiende a estudiantes con espectro autista, espacio en donde se desempeña como docente uno de los autores de este articulo. Esta motivación educativa es llevada al terreno de la investigación e intenta aportar a la comprensión de las interacciones comunicativas de un joven del espectro autista y así interpretar los significados creados en un determinado contexto escolar, con el fin de mejorar la Calidad de Vida de dicho estudiante.

El segundo factor a considerar es que este estudio se enmarca en un proyecto de investigación mayor (Fondecyt 1130684), el cual aborda las interacciones comunicativas en el aula, desde la perspectiva de la Semiótica Social y la Multimodalidad. Lo cual implica que esta perspectiva teórica permite poner en un lugar central a todos los modos semióticos que se utilizan en la comunicación y representación, y no solo al lingüístico (Kress y van Leeuwen, 1996). 
En consecuencia, esta investigación tiene como objetivo describir los recursos comunicativos que utiliza una persona del espectro autista en su contexto escolar. La descripción se efectúa con un doble lente: un lente pedagógico -el cual atiende a las necesidades de apoyo que requiere el estudiante con el fin de mejorar su Calidad de Vida (Schalock, 2011) - y uno semiótico - el cual identifica y valora los recursos comunicativos en contextos situados y los significados creados a través de ellos-. Ambos enfoques fundamentan teóricamente la investigación y orientan los resultados y conclusiones.

\section{Marco Teórico}

\subsection{Condición de espectro autista y paradigma inclusivo}

Con el fin de fundar las bases para poder observar y describir la comunicación de una persona que presenta rasgos del espectro autista, se pone en común algunos conceptos y terminologías que se utilizan a lo largo del artículo. Primero es necesario comenzar por la condición del espectro autista.

El autismo -descrito en sus inicios por Leo Kanner en 1943- se define desde la psicología y neurología como un trastorno del neurodesarrollo que afecta la interacción y la comunicación y que se caracteriza por presentar conductas e intereses limitados y repetitivos (Belinchón, 2010). A partir de esta definición más bien general, se pueden apreciar diferencias entre quienes presentan el espectro autista dependiendo de los contextos sociales donde se desempeña y las características y potencialidades personales (Riviere, 1990), estas se ven reflejadas en diagnósticos variados, por eso se habla de espectro. Por ejemplo, las personas del espectro autista pueden presentar características como discapacidad intelectual (en varios niveles o grados), presencia o ausencia de lenguaje verbal, respuesta exacerbada a algunos estímulos sensoperceptivos, entre otros.

Respecto a las características personales de cada niño o adulto del espectro, se consideran tres criterios fundamentales que configuran su diagnóstico: 1. alteraciones cualitativas en la interacción social recíproca; 2 . alteraciones en la comunicación verbal y no verbal; y 3. presencia de patrones restrictivos, repetitivos y estereotipados de la conducta (Wing, 1997). La triada de Wing se puede expresar en diversos grados en cada uno de los rasgos mencionados, sin embargo, es la descripción común para todas las personas del espectro.

Específicamente en cuanto al lenguaje, entre sus características se ha descrito un retraso en el desarrollo lingüístico e incluso ausencia de lenguaje en algunas personas del 
espectro autista. Cuando desarrollan lenguaje suelen hacer selección léxica y/o sintáctica poco común; frecuentemente presentan ecolalia, tendencia a repetir de forma literal lo que oyen y también, en general comprenden el lenguaje de forma muy literal. Para quienes no han desarrollado completamente el lenguaje se describen vocalizaciones sin lenguaje 0 también lenguaje hablado, pero sin carácter comunicativo (Martos y Riviere, 2001).

Se debe considerar que las concepciones y formas de establecer las interacciones entre personas dentro del espectro autista y sus interlocutores se encuentran determinadas en amplia medida por las representaciones sobre la condición del espectro autista que han desarrollado los interlocutores, estas estructuran las relaciones sociales y los intercambios comunicativos ya que funcionan como estereotipos (Riviere y Núñez, 1996). Tal como dice Quirós (2006) al partir de estos estereotipos, quienes interactúan con las personas dentro del espectro autista generan un prejuicio respecto de las conductas o emociones de ellos. Estas atribuciones pueden ser erróneas lo que afecta las expectativas sobre lo que las personas del espectro autista pueden o no pueden hacer, impactando en los apoyos a las potencialidades y necesidades que se podrán diseñar en el contexto educativo.

A través del tiempo los paradigmas educativos se han ido modificando de acuerdo con la concepción de ser humano y las tradiciones epistemológicas (Cabrera, 2012). En los años noventa se tenía como orientación educativa las prácticas integradoras, donde se identifican falencias en una persona, las cuales tienen que ser mejoradas o potenciadas para integrarse en la sociedad donde se desempeña. Esta visión se observa en los grupos interdisciplinarios de orientación clínica, los cuales apuntan a desarrollar las habilidades que se identifican como "descendidas" dentro de un marco de normalidad. Sin embargo, en la actualidad la visión del ser humano como una naturaleza única se ha debilitado, pasando a una definición interpretativa, lo cual genera diversas concepciones de lo que significa ser humano (Tamarit, 2005). Esto implica un cambio hacia la Perspectiva Inclusiva en la educación, lo que se relaciona con la valoración humanista de la persona como tal, y por ende no se trata de mejorar sujetos que carecen de una habilidad o función para "completarlos", sino mas bien, se pretende adaptar o cambiar la cultura, derribando barreras y otorgando apoyos que aprecien la diversidad de formas de vivir y de ser (Cabrera, 2012).

Incluso, en la última edición del DSM-V (2013), proponen un nuevo método de evaluación a utilizar, eliminando límites diagnósticos como el Trastorno de Asperger, trastorno de Rett y las otras categorías. De esta forma, la diversidad de características identificadas se encuentran descritas de manera más integral, centrando la clasificación en 
los diferentes niveles de apoyo que requiera la persona de acuerdo a su funcionamiento en la interacción con su entorno.

Teniendo en cuenta las características previamente descritas, incluyendo el contexto y la postura educativa desde donde se posiciona este artículo, se presentan variados desafíos a quienes interactúan con personas del espectro autista, esto incluye desde los profesionales que los atienden hasta sus familiares más cercanos. Uno de estos desafíos es la interpretación de los comportamientos y de las maneras de comunicarse en distintos contextos, las cuales son percibidas con frecuencia como peculiares y/o disruptivas por el interlocutor. Como plantea Riviere (1990) respecto al comportamiento comunicativo de las personas con dentro del espectro autista, estos son muy comunicativos, sin embargo, lo expresan de manera particular.

A partir de estas peculiaridades podemos tomar dos opciones $u$ orientaciones frente al comportamiento mencionado, una de ellas es atribuir una dificultad al estudiante respecto a sus habilidades comunicativas o de comportamiento, lo que corresponde a una postura del paradigma de la integración. La otra opción es valorar sus peculiaridades comunicativas y abrir espacios en los contextos de desempeño de cada persona del espectro autista, interpretando los significados comunicados, identificando necesidades y otorgando apoyos (Shalock, 2011) con el fin de mejorar su Calidad de Vida. Esta última correspondería a una postura orientada por el paradigma inclusivo. Ahora bien, una manera de abordar teórica y metodológicamente la interpretación de los recursos comunicativos de una persona del espectro autista, teniendo en cuenta su individualidad y particularidad en sus recursos para comunicar, es la Semiótica Social y la Lingüística Sistémico Funcional, las cuales abordaremos a continuación.

\subsection{Semiótica Social y Lingüística Sistémico Funcional: miradas contextualizadas}

Para abordar el fenómeno comunicativo, en este estudio se consideran los aportes de la Semiótica Social, la Lingüística Sistémico Funcional (en adelante LSF) y el enfoque Multimodal sobre la comunicación. Esta selección resulta apropiada para observar la comunicación de manera amplia y contextual, ya que plantean una teoría funcional del lenguaje y de los otros sistemas semióticos, además de ofrecer aspectos metodológicos para el análisis de los diversos intercambios de significados en una cultura. 
La Semiótica Social considera que los distintos sistemas semióticos son productos culturales que surgen y se modifican a la luz de las necesidades de representación y comunicación de los distintos grupos sociales (Halliday, 1978; Hodge y Kress, 1988). Desde esta teoría es posible preguntarse tanto por los significados que intercambian las personas en determinados contextos así como por los recursos que utilizan para hacerlo. Halliday (1978) en su teoría LSF, propone una serie de conceptos articulados para interpretar los fenómenos semánticos de manera contextualizada, es decir, en una relación interdependiente entre texto y contexto, cuyo mayor desarrollo se encuentra en relación al sistema lingüístico. Los sistemas semióticos o de significado se presentan como un conjunto potencial de recursos para significar, es decir, sistemas que han sido convencionalizados en las distintas comunidades para construir significados para interactuar con otros y con el mundo y con ello satisfacer necesidades comunicativas (Halliday, 1983). Ontogenéticamente, el uso de los signos puestos en juego en la creación de significado o semiosis son importantes, ya que se relacionan con el proceso mediante el cual las personas interiorizan las formas de ver el mundo de su comunidad a partir de los signos como herramientas culturales (Vigotsky, 1998). En este sentido, la propuesta de la Semiótica Social es complementaria con el enfoque Sociocultural, el cual es ampliamente conocido en el ámbito educativo a través de la teoría vigotskyana.

Si nos enfocamos en la semiosis como aspecto esencial para comprender al ser humano en cada acto de comunicación lo nuclear es lo semántico, esto es: el significado que se construye e intercambia con otros. La Semiótica Social y la LSF entienden el significado desde tres perspectivas (Gutiérrez, 2011). La primera de ellas es el significado como elección, es decir, a partir de los sistemas semióticos disponibles en su cultura, la persona que crea significado, elige cuáles unidades de significado usar y combinar para construir un significado mayor en un texto. Este acto de elección de un recurso en vez de otro, conlleva significado en sí mismo, estaría motivado por el contexto y a la vez, los significados construirían el contexto en la interacción semiótica.

En la segunda perspectiva, el significado se entiende como variación, es decir, cada significado es relativamente estable en una comunidad pero no es fijo ya que se configura sobre manifestaciones concretas en situaciones determinadas: el significado cambia o varía dependiendo del contexto. La LSF se preocupa de algunos rasgos que se presentan regularmente en los contextos y que se asocian a las elecciones que se hacen desde el sistema. Por ejemplo, a la hora de comprar fruta, generalmente elegimos los mismos 
recursos para un intercambio de significados bastante estable. El registro es el sustento teórico que permite dar cuenta de la descripción del contexto de situación en referencia a tres variables:1) tenor, quiénes se comunican y cuál es su relación; 2) campo, en qué actividad se encuentran y sobre qué tema se comunican; y 3) modo, cuáles son los recursos semióticos disponibles para comunicarse y cómo los combinan en un texto (Halliday, 1978).

En la tercera perspectiva el significado se comprende de manera central como función. A partir de la dependencia contextual del significado Halliday (1978) plantea la hipótesis metafuncional: cada sistema semiótico permite expresar tres funciones simultáneamente: la función interpersonal, la ideacional y la textual.

La metafunción interpersonal, también denominada comunicativa (Kress y van Leeuwen, 1996), enactúa las relaciones sociales y personales con las otras personas del entorno donde se organiza la realidad social de las personas con las cuales interactuamos. La LSF permite acercarnos a la interacción desde una perspectiva semántica, ya que cada vez que interactuamos con alguien, independientemente de las características de los interlocutores, lo hacemos usando un sistema semiótico que permite establecer una relación entre quien crea el significado y toma su turno en la comunicación, y la persona que lo interpreta probablemente tomará su turno a continuación (Eggins, 2004).

Para poder comprender mejor cómo ocurre el intercambio de significados es necesario considerar que mientras que tomamos turnos en la comunicación adoptamos diferentes roles en el intercambio de significados. Tal como sintetiza la Tabla 1, e partir del uso de los diversos recursos semióticos disponibles para el intercambio de significados, la teoría LSF describe cuatro acciones comunicativas centrales: afirmaciones, preguntas, órdenes y ofrecimientos, las cuales tienen que ver con el rol que asumimos en la comunicación (ofrecer/demandar) así como con el objeto de intercambio (información / bienes y servicios) (Halliday \& Matthiessen, 1994). Los bienes y servicios corresponden a objetos y acciones, es decir, en un intercambio comunicativo además de información, se puede dar o pedir objetos y acciones que no necesariamente son lingüísticas, pero que completan el intercambio comunicativo. Por ejemplo: puedo estirar la mano para pedir pan (demanda de un bien) y mi interlocutor me lo puede pasar sin ninguna emisión lingüística (ofrecimiento de un bien). La metafunción interpersonal también permite expresar cuán seguros nos sentimos de lo que decimos, cómo valoramos a las personas y sus actos, cual es nuestra apreciación personal de las cosas o fenómenos, entre otros; a través de la creación de significado o semiosis (Oteiza, 2006; Moss, 2010). 
Tabla 1: Roles del discurso

\begin{tabular}{|l|l|l|}
\hline \multicolumn{3}{|c|}{ Intercambio de significado } \\
\hline Rol del discurso & Información & Bienes y servicios \\
\hline Ofrecer & Afirmación & Ofrecimiento \\
\hline Demandar & Pregunta & Orden \\
\hline \multicolumn{2}{|l|}{ Fuente: Tabla Roles del discurso e intercambios en la interacción (Halliday 1994: 69) }
\end{tabular}

En cuanto a la metafunción ideacional, denominada por Kress y van Leeuwen (1996) como función representacional, supone que la totalidad de la experiencia humana sería potencialmente posible de ser convertida en significado, es decir, todo podría representarse a través de algún recurso para significar. Es por esto que los diversos recursos constituirían sistemas de significados que ofrecen una teoría de la experiencia humana. El significado ideacional tiene que ver con la representación de quién hace qué, a quién, cuándo, dónde, por qué y cómo; reflejada cuando utilizamos algún recurso semiótico para representar nuestras experiencias acerca del mundo, para describir eventos, entre otros (Eggins y Martin, 2003; Oteiza, 2006).

Finalmente, la metafunción textual es la función organizadora de los recursos semióticos en un texto, la que pone en relación los distintos significados en un significado mayor. Por lo tanto, es la función que habilita la creación de significados ideacionales e interpersonales, formando un texto coherente para el interlocutor. Ontogenéticamente es la última metafunción del lenguaje que se desarrolla (Halliday, 1978).

El último sustento teórico que se aborda para completar la mirada sobre la comunicación es la multimodalidad. El enfoque multimodal sobre la comunicación, en oposición al monomodal, se caracteriza por considerar, por una parte, la creación de significados en un contexto y, por otra, pone su mirada más allá del recurso lingüístico. La multimodalidad es una perspectiva que permite comprender los escenarios comunicativos de manera compleja, ya que considera que cada uno de los modos semióticos puestos en marcha para la semiosis o creación de significado aporta de manera diferente al significado mayor (Kress y van Leeuwen, 2001). De esta manera, todo texto es multimodal, en la medida en que se utilicen diversos modos semióticos (habla, gestos, dibujos, escritura, entre otros.) en el diseño de un producto o evento semiótico (Kress, 2010).

La multimodalidad como enfoque semiótico permite, entonces, observar la interacción y describir la combinación de recursos semióticos bajo el supuesto de que todos ellos aportan a un intercambio de significados, ya sea una palabra, una expresión facial o corporal, cada una de ellas se pondría en marcha con un fin comunicativo. 
Entre los antecedentes respecto de un abordaje semiótico de la comunicación de personas del espectro autista, destaca el estudio de Dreyfus (2006). A continuación, se sintetizan algunos de sus hallazgos.

\subsection{Diversas formas de comunicar}

La presente investigación está orientada por el trabajo de Dreyfus (2006; 2011) quien analizó e interpretó las interacciones comunicativas de su hijo Bodhi - quien posee como diagnóstico una discapacidad intelectual y comunicativa severa. Para esto utilizó el marco teórico de la Semiótica Social y la LSF.

Dreyfus (2006) plantea que existen "malos entendidos" en los interacciones comunicativas entre Bodhi y las personas con quienes realiza estos intercambios de significados. Los malentendidos son frecuentes entre Bodhi y las personas que no se relacionan frecuentemente con él y que, por lo tanto, no conocen sus modos de comunicación. No obstante, estas situaciones también ocurren con las personas cercanas, como los son sus familiares. Si bien en el caso de Bodhi, las interacciones comunicativas son generalmente no verbales, al contrario de lo que se podría suponer, él comprende lo que le comunican y es muy expresivo, sin embargo, utiliza otros modos para comunicar. Esto genera frecuentemente una interpretación errónea de los modos comunicativos o semióticos que pone en marcha Bodhi para crear significado.

Lo particular es, que según este estudio, son los interlocutores quienes malinterpretan o malentienden a Bodhi y no al revés, es decir, Bodhi comprende e interactúa en el intercambio de significados pero cuando él inicia la interacción o entrega información o bienes y servicios sus interlocutores no interpretan adecuadamente sus expresiones. De esta situación, y a partir del marco teórico de la inclusión, es posible apreciar que son los interlocutores los que podrían abrir su propio espectro comunicativo, para participar adecuadamente en el intercambio de significados.

Dreyfus (2011) plantea que, en general, las personas con discapacidad intelectual severa se comunican de manera muy ambigua, lo cual propicia estos malentendidos que tienen como consecuencia frustración y comportamiento agresivo. Esboza también que la LSF es un medio útil para clasificar o identificar los tipos de malentendidos o modos que utiliza Bodhi.

La autora destaca que existen dos particularidades en la forma de comunicación de Bodhi: la primera se relaciona con el uso de los modos o recursos comunicativos puesto que 
cuando Bodhi se comunica utiliza un solo modo por intercambio. Es decir, se podría plantear que las interacciones de Bodhi son mono modales, no combina más de un modo por toma de turno; La segunda característica se relaciona con el significado contextualizado, es así como un mismo modo de expresión puede tener distintas funciones en su discurso y esto sería lo que genera gran ambigüedad por las personas que no ponen atención a los factores contextuales de la interacción.

Por ejemplo, en una situación en que Bodhi quiere realizar su actividad favorita que es subir una escalera, él apunta la imagen de una escalera demandando un servicio: quiero que me lleves para allá, y su cuidador lo lleva a la escalera. A pesar de esto, él sigue apuntando la imagen de la escalera, ya que ahora estaría ofreciendo información que su cuidador no logra interpretar, ya que usa el mismo recurso para otra función. Entonces, cuando Bodhi demanda un servicio simultáneamente demanda información de su compañero (Dreyfus, 2011).

De esta manera, resulta importante identificar los modos que utiliza Bodhi (o la persona a la cual se está observando) e identificar claramente si este modo es utilizado como oferta o demanda de información o bienes y servicios, para lo cual, al igual como lo realiza Dreyfus, es necesario identificar los recursos que utiliza la persona para comunicarse. A continuación se presenta la metodología de investigación, la cual contiene los insumos de la LSF de manera práctica y el contexto en donde se desempeña el presente trabajo.

\section{Metodología}

Esta investigación es parte de un proyecto mayor el cual aborda la alfabetización semiótica en la escuela y su mediación, a través de la descripción multimodal de las interacciones semióticas de los estudiantes y de los profesores (Proyecto Fondecyt 1130684). Dentro de los objetivos planteados, este artículo apunta a describir los recursos semióticos utilizados por un alumno del espectro autista de una escuela de Educación Especial de la comuna de Viña del Mar en Chile.

Desde una perspectiva cualitativa, abordamos esta investigación en torno a situaciones comunicativas de interacción escolar en su entorno natural, rescatando la singularidad que la configura, partir de intentar interpretar los acontecimientos, acciones, normas, valores, entre otros, desde la perspectiva de las personas que están siendo estudiadas (Mella, 1998).

El enfoque teórico metodológico para la descripción de la comunicación en un joven con Espectro Autista y escasos recursos verbales, corresponde a la Semiótica Social (Hodge 
y Kress, 1988; Halliday, 1978). Este enfoque relaciona los significados con el contexto en dos niveles: 1) contexto social y cultural; 2) contexto situacional o registro (campo, tenor y modo).

El diseño corresponde a un estudio de caso único centrado en la interacción del estudiante en el contexto escolar. Para esto se realizaron grabaciones audiovisuales en el último período de clases (11:40 a 13:00 hrs.) durante tres días en cada una de cuatro semanas consecutivas. Con esto se constituyó un corpus audiovisual de 13 sesiones de grabación.

El análisis de los datos se lleva a cabo desde el Análisis Multimodal del Discurso (Kress y van Leeuwen, 1996) y se utilizan como herramientas heurísticas para explorar el corpus audiovisual la Gramática del Diseño Visual de Kress y van Leeuwen (1996) y las metafunciones de Halliday (1978). El análisis se concentra en la metafunción interpersonal ya que esta es la que permite dar cuenta de las interacciones e intercambio de significados.

Para el análisis se revisaron los videos con detalle, con el fin de realizar una interpretación y registrar los recursos semióticos utilizados por el estudiante en el desarrollo de cada sesión en la búsqueda de regularidades. Se utilizó una tabla de análisis para describir el despliegue temporal de los significados dando muestra gráfica del orden en que se presentan los recursos para comunicarse durante el periodo de clases, permitiendo analizar la frecuencia de aparición en relación al contexto. A continuación se puede observar la Tabla 2 utilizada para realizar los análisis de los videos:

Tabla 2: análisis del corpus audiovisual

\begin{tabular}{|l|l|l|l|l|l|}
\hline $\begin{array}{c}\text { Período de } \\
\text { grabación }\end{array}$ & $\begin{array}{c}\text { Despliegue } \\
\text { temporal }\end{array}$ & Campo & Tenor & Modo & Descripción de la interacción \\
\hline & & & & & \\
\hline & & & & & \\
\hline
\end{tabular}

Fuente: Adaptada de Echeverría, González, Guajardo, Martínez y Rodríguez (2011)

El instrumento utilizado para el análisis multimodal de los videos (tabla 2) consta de 6 columnas definidas operacionalmente de izquierda a derecha de la siguiente manera:

1) Período de grabación: Período del día en que se realiza la grabación, consigna la hora de grabación y la asignatura o actividad curricular observada.

2) Despliegue temporal: Se indican los minutos y segundos en que se inicia y finaliza cada intercambio de significado durante la grabación. 
3) Campo: Actividad y tema que configura el contexto de situación ¿de qué hablan o se comunican?

4) Tenor: Roles de cada persona durante las interacciones que configura el contexto de situación (pide/da; información/ bienes y servicios) ¿en qué rol se encuentra cada interlocutor?

5) Modo: tipo de recurso utilizado para la interacción comunicativa que configura el contexto de situación (palabra hablada, gesto, acción,...) ¿Qué recursos se usan para significar?

6) Descripción de la interacción: Descripción contextual de las interacciones, son las interpretaciones de los analistas.

A partir de las 13 sesiones analizadas a través de la Tabla 2 de análisis del corpus audiovisual, se buscan regularidades en los recursos para construir significado en las interacciones cotidianas. Posteriormente, se construye un repertorio de recursos semióticos utilizados en el estudio de casos para la metafunción interpersonal, en la tabla 3 se aprecia la oferta (dar en la interacción) y demanda (solicitar en la interacción) de bienes (objetos materiales), servicios (acciones) e información.

Tabla 3: Roles del discurso

\begin{tabular}{|l|l|l|}
\hline \multicolumn{3}{|c|}{ Intercambio de significado } \\
\hline Rol del discurso & Información & Bienes y servicios \\
\hline Ofrecer & Afirmación & Ofrecimiento \\
\hline Demandar & Pregunta & Orden \\
\hline
\end{tabular}

Fuente: Tabla Roles del discurso e intercambios en la interacción (Halliday 1994, p. 69)

Teniendo en cuenta los insumos teórico-metodológicos previamente mencionados, a continuación se presenta los hallazgos obtenidos.

\section{Hallazgos}

En esta sección se mostrará en primer lugar el repertorio de recursos que utiliza el estudiante para significar en el contexto escolar. En segundo lugar, se profundizará en un recurso de significación particular, el cual consiste en "tirarse al suelo", para luego finalizar con una interpretación de este recurso a partir del contexto de situación en el cual ocurre la interacción. Cabe recordar, que el análisis en cuestión se enfoca en la metafunción interpersonal, a través de la demanda u oferta de bienes, servicios o información. 


\subsection{Repertorio o abanico de recursos semióticos}

A través de la revisión de las interacciones del estudiante con su entorno escolar, se identifican los siguientes recursos para significar en el ámbito de la metafunción interpersonal, los cuales están organizados en la Tabla 4 que se muestra a continuación:

Las columnas de la Tabla 4 muestran el abanico de recursos semióticos que utiliza el estudiante en el contexto escolar, dividido en la demanda - aquellos que solicita - y la oferta aquello que entrega - en las interacciones comunicativas.

En un primer lugar, el análisis permite dar cuenta de los recursos que de forma global constituyen el repertorio comunicativo del estudio de caso. Por un lado, los recursos que el estudiante utiliza para la demanda, corresponden a un total de ocho modos semióticos: contacto visual, tirarse al suelo, gritar, escupir, hurgar la mochila, tomar objetos, mirar por la ventana y verbalizar algunas palabras como "mamá", "chao” y “Jim de la Luna”. Por otro lado, los recursos utilizados para la oferta son muy diversos y numerosos entre los que destacan con más frecuencia: pellizcar, reír, llorar, aplaudir, agitar cabeza, golpear objetos, huir, gesticular adiós con su mano, entre otros (ver Tabla 4).

Tabla 4: Recursos semióticos para la comunicación

\begin{tabular}{|c|c|c|c|c|c|c|c|c|}
\hline \multicolumn{9}{|c|}{ ABANICO DE RECURSOS SEMIÓTICOS } \\
\hline \multicolumn{4}{|c|}{ DEMANDA } & \multicolumn{5}{|c|}{ OFERTA } \\
\hline \multirow[t]{2}{*}{$\begin{array}{l}\text { Contacto } \\
\text { visual }\end{array}$} & \multirow[t]{2}{*}{$\begin{array}{l}\text { Tirarse al } \\
\text { suelo }\end{array}$} & \multirow[t]{2}{*}{$\begin{array}{l}\text { Imitación } \\
\text { de llanto }\end{array}$} & \multirow[t]{2}{*}{ Escupir } & Agachar la cabeza & $\begin{array}{c}\text { Evitar sentarse en } \\
\text { su silla }\end{array}$ & Pellizcar & Aplaudir & Reír \\
\hline & & & & $\begin{array}{l}\text { Levantarse de la } \\
\text { silla }\end{array}$ & Agitar cabeza & Sonreír & $\begin{array}{c}\text { Verbalizar } \\
\text { (hola) }\end{array}$ & $\begin{array}{c}\text { Gesticula } \\
\text { adiós } \\
\text { con su } \\
\text { mano }\end{array}$ \\
\hline \multirow[t]{2}{*}{$\begin{array}{l}\text { Hurgar } \\
\text { mochila }\end{array}$} & \multirow[t]{2}{*}{$\begin{array}{l}\text { Tomar } \\
\text { objetos }\end{array}$} & \multirow{2}{*}{$\begin{array}{l}\text { Verbalizar } \\
\text { (mamá, } \\
\text { chao, Jim } \\
\text { de la Luna) }\end{array}$} & \multirow[t]{2}{*}{$\begin{array}{l}\text { Mira por } \\
\text { la } \\
\text { ventana }\end{array}$} & Tocar su entrecejo & $\begin{array}{l}\text { Tomar objeto de } \\
\text { compañero }\end{array}$ & $\begin{array}{l}\text { Golpear } \\
\text { objetos }\end{array}$ & Huir & $\begin{array}{l}\text { Respond } \\
\text { e al } \\
\text { llamado }\end{array}$ \\
\hline & & & & Escupe & Imitación de llanto & $\begin{array}{c}\text { Se pone de } \\
\text { pie }\end{array}$ & & \\
\hline
\end{tabular}

Fuente: creada por los autores (2013)

A primera vista se logra apreciar que los recursos que el estudiante utiliza para la demanda, es decir, para solicitar información, bienes y servicios en sus interacciones comunicativas, son menores en cantidad en relación a los recursos utilizados en la oferta. Cabe mencionar que respecto a esta diferencia entre la oferta y la demanda hay dos aclaraciones que realizar, que se desprenden de la observación del contexto escolar. Primero, si bien los recursos para la demanda son menores en cantidad a la oferta, el estudiante demanda de manera consistente y reiterada, siendo las instancias de demanda notoriamente más frecuentes que la oferta durante los intercambios comunicativos 
observados. Esta característica es consistente con la descripción del Espectro Autista referido al lenguaje instrumental y los intereses restringidos del estudiante, lo que provoca mayor interés en demandar los objetos u acciones que desea, a través de los adultos que le rodean.

La segunda aclaración en relación con la cantidad de recursos implementados para la oferta y la demanda, esta da cuenta de una característica del repertorio comunicativo del estudiante. Por una parte, la menor cantidad de recursos para la demanda, en este caso, da cuenta del nivel de estabilización de los recursos que utiliza el estudiante. En cada contexto de situación el estudiante utiliza ciertos recursos para demandar información, bienes o servicios, es decir, estos recursos son ya parte estable de su repertorio semiótico y puede diferenciar entre sus funciones. Por otra parte, la oferta de bienes, servicios, o información, si bien presenta mayor diversidad de recursos, la mayor parte de estos son utilizados de forma aleatoria y espontánea, lo que implica una menor estabilización de los recursos y menor diferenciación de sus funciones, y para los interlocutores una mayor dificultad para interpretar los significados comunicados.

Estos diferentes grados de estabilización de los recursos semióticos y comunicativos coinciden con las investigaciones realizadas por Bates y equipo (1979) respecto al desarrollo del lenguaje. Su propuesta describe cómo los niños en la etapa pre-lingüística estabilizan las funciones comunicativas a través del uso de recursos semióticos específicos para alcanzar objetivos concretos. Estas funciones se vuelven cada vez más consistentes en el tiempo, lo que denomina formas comunicativas consistentes. Si consideramos, además, las características descritas respecto a los intereses restringidos de las personas dentro del Espectro Autista (Wing, 1997), podríamos decir que la demanda, al estar en función de los intereses restringidos, podría facilitar esta diferenciación funcional entre los recursos comunicativos.

Por ejemplo, dado que dentro de los intereses restringidos del estudiante se encuentra una botella de bebida, un peluche y un dibujo de "Jim de la Luna", el estudiante utiliza el recurso de "imitación de llanto" de forma estabilizada, cada vez que pide alguno de los objetos mencionados. Este recurso se caracteriza por una mirada intensa al interlocutor, una expresión facial de angustia y un llanto fingido con una tonalidad e intensidad específica, que va aumentando en la medida que demora la respuesta a su demanda. Este recurso deja de ser utilizado en el momento en que el estudiante recupera el objeto de demanda. 
En cuanto a la oferta, los recursos "tocarse el entrecejo" y "verbalizar y/o gesticular Chao" son usados consistentemente para informar que está enojado y para informar que quiere poner fin a la interacción, respectivamente. Los recursos restantes son utilizados de formas indistintas, constituyéndose como funciones comunicativas menos estabilizadas. Siguiendo este mismo razonamiento, los recursos utilizados para la oferta se encontrarían en un nivel menos estabilizado de sus funciones, requiriendo por parte del interlocutor de una interpretación más contextualizada y un mayor conocimiento de la persona con autismo que en las situaciones de demanda.

Para describir con mayor detalle los objetos de intercambio, es posible apreciar en la Tabla 5 la subdivisión de los recursos utilizados para la oferta y demanda en bienes, servicios e información, los cuales corresponden respectivamente a la demanda u oferta de objetos, acciones o informaciones que el estudiante utiliza en las interacciones en contexto escolar. En la Tabla 5 se observan que recursos utiliza para tal o cual objeto comunicativo.

Tabla 5: Desglose de recursos semióticos

\begin{tabular}{|c|c|c|c|c|c|}
\hline \multicolumn{6}{|c|}{ DESGLOSE DE RECURSOS SEMIÓTICOS } \\
\hline \multicolumn{3}{|c|}{ Demanda } & \multicolumn{3}{|c|}{ Oferta } \\
\hline Bienes & Servicios & Información & Bienes & Servicios & Información \\
\hline Tirarse al suelo & Tirarse al suelo & Tirarse al suelo & & Huir & Golpear objeto \\
\hline $\begin{array}{l}\text { Verbalizar (Jim de } \\
\text { la Luna) }\end{array}$ & 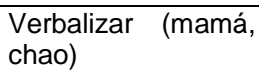 & $\begin{array}{l}\text { Verbalizar } \\
\text { (mamá) }\end{array}$ & & Escupir & Tocar entrecejo \\
\hline Hurgar mochila & Contacto visual & & & Verbalizar (hola) & Reír \\
\hline Llanto fingido & Mira por la ventana & & & $\begin{array}{l}\text { Evitar sentarse } \\
\text { en su silla }\end{array}$ & Agitar cabeza \\
\hline \multirow[t]{11}{*}{ Tomar objetos } & Escupir & & & $\begin{array}{l}\text { Levantarse de la } \\
\text { silla }\end{array}$ & $\begin{array}{l}\text { Agachar la } \\
\text { cabeza }\end{array}$ \\
\hline & & & & \multirow{2}{*}{$\begin{array}{l}\text { Tomar objeto de } \\
\text { compañero }\end{array}$} & Pellizcar \\
\hline & & & & & Sonreír \\
\hline & & & & $\begin{array}{l}\text { Gesticula adiós } \\
\text { con su mano }\end{array}$ & $\begin{array}{ll}\text {-Tocar } & \text { su } \\
\text { entrecejo } & \\
\end{array}$ \\
\hline & & & & Se pone de pie & -Golpear objetos \\
\hline & & & & & Aplaudir \\
\hline & & & & & Verbalizar (hola) \\
\hline & & & & & Reír \\
\hline & & & & & $\begin{array}{l}\text { Responde } \\
\text { llamado }\end{array}$ \\
\hline & & & & & Escupe \\
\hline & & & & & Llora \\
\hline
\end{tabular}

Fuente: creada por los autores (2013)

Un elemento importante a destacar a partir del análisis de la Tabla $N^{\circ} 5$, es que al igual como señala Dreyfus (2011) a partir de su investigación, estos resultados de un estudio de caso demuestran que esta personas dentro del Espectro Autista o con severa discapacidad comunicativa también utiliza recursos comunicativos poco consistentes, emplean el mismo 
recurso en diversos contextos y para significar en diferentes situaciones, generando ambigüedad en la interpretación de los significados por parte de los interlocutores. Así, en el caso de la demanda, tenemos el recurso de "tirarse al suelo", el cual se repite tanto en demanda de información, bienes y servicios ¿Cómo interpretar estos significados que nos resultan ambiguos en la interacción con personas autismo? Para hacer una interpretación adecuada de la interacción comunicativa y de la función del recurso utilizado es necesario analizarlo desde su contexto de situación, es decir, el recurso utilizado significará tal o cual cosa, dependiendo del contexto situacional donde el joven use este recurso.

Por ejemplo, el "tirarse al suelo" significará una demanda de información en el contexto de almuerzo en el comedor de la escuela, en cambio, significará demanda de un servicio durante el periodo de clases en el aula. Este recurso de "tirarse al suelo" requiere un análisis situado para realizar una interpretación adecuada. Sumado a esto, el modo semiótico se presenta por lo general de forma enérgica y repentina, lo que genera desconcierto en el entorno. A continuación, se profundizará en este recurso en particular.

\subsection{Análisis del recurso semiótico "Tirarse al suelo"}

Si bien uno de los resultados de los análisis es el repertorio de recursos semióticos, también es posible mostrar un análisis en profundidad de uno de los recursos que utiliza el estudiante para significar, este recurso es el "tirarse al suelo". El recurso de "tirarse al suelo" posee la particularidad de expresarse en diferentes situaciones comunicativas. El estudiante hace uso de este recurso durante todo el periodo de clases, mostrando un aumento en frecuencia e intensidad de la conducta durante el último periodo, cuando se acerca la hora de salida de la escuela, el cual corresponde al almuerzo. El estudiante utiliza el recurso, ya sea en la sala de clases durante la asignatura de expresión artística o en la sala de psicomotricidad durante el taller de relajación, y luego en el comedor durante el almuerzo propiamente tal.

A continuación, se muestra en la Tabla 6 el registro de las interacciones de estas tres situaciones comunicativas en las que el estudiante utiliza este recurso. Recordemos que el registro corresponde a las tres variables campo, tenor y modo que describen y configuran la situación comunicativa. 
Tabla 6: Registro de uso general del recurso "tirarse al suelo"

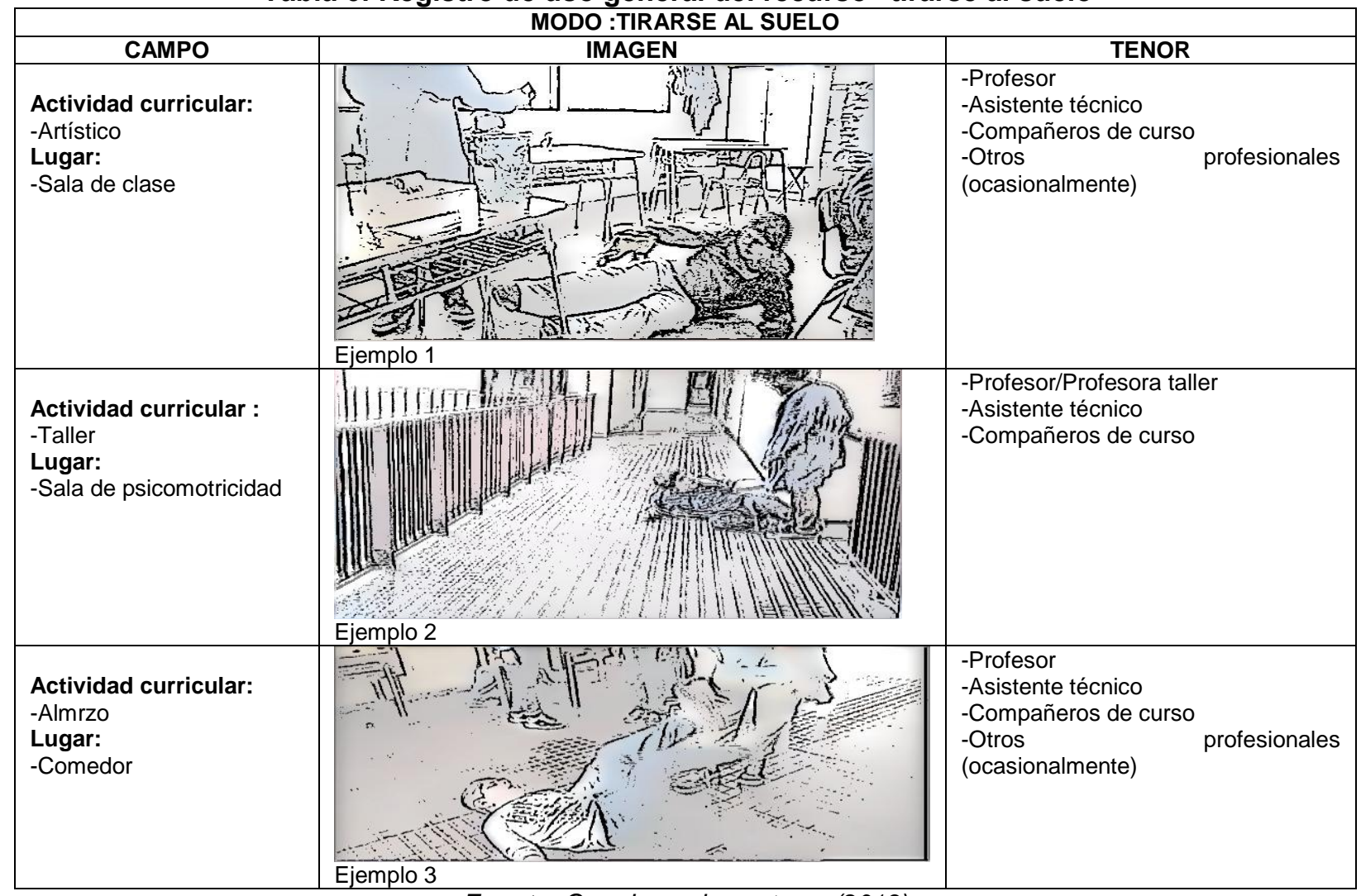

Fuente: Creada por los autores (2013)

En la Tabla 6 es posible apreciar el despliegue del recurso "tirarse al suelo" a través de diferentes registros. El recurso en cuestión se expresa en los tres contextos de CAMPO: actividades curriculares de artística, taller y almuerzo, con un TENOR en donde participan el profesor, la asistente técnica del curso y compañeros del curso. La interpretación general de este recurso dice relación con comenzar/finalizar una actividad. Sin embargo, esta interpretación sigue siendo demasiado general para impactar en la Calidad de Vida del estudiante, para lo cual es necesario especificar en contexto de desempeño, es decir, generar una interpretación particular en el contexto de situación donde emerge el recurso utilizado.

Para esto se ha seleccionado el contexto de "Comedor" en la actividad de "Almuerzo" con la presencia de "Compañeros de curso", "Otros compañeros", "Profesor y asistente técnico". Es necesario tener en cuenta que la actividad de almorzar es la última a realizar antes de la salida de la escuela, y es justamente, en este contexto, en donde el recurso de "tirarse al suelo", se interpreta por la comunidad educativa solamente como una conducta 
disruptiva, y no como un recurso comunicativo. A continuación en la Tabla 7 se aprecia el contexto de situación del análisis del recurso "tirarse al suelo".

Tabla 7: Registro del recurso "tirarse al suelo" en contexto de almuerzo

\begin{tabular}{|c|c|c|}
\hline CAMPO & TENOR & MODO \\
\hline $\begin{array}{c}\text { Actividad: } \\
\text { Comedor/Almuerzo } \\
\text { (12:30 hrs, ultima actividad antes de } \\
\text { salida de la escuela) }\end{array}$ & $\begin{array}{c}\text { Profesores } \\
\text { Compañeros de curso } \\
\text { Compañeros de escuela }\end{array}$ & Tirarse al suelo \\
\hline
\end{tabular}

Fuente: Creada por los autores (2013)

Es necesario recordar que el análisis del significado del "tirarse al suelo" es realizado siempre en su contexto, por lo cual, el significado del recurso está supeditado a su contexto de situación o registro (campo- tenor- modo), esto nos puede dar luces de que otros significados a partir del mismo recurso se pueden configurar en otras situaciones, pero que no necesariamente se podrá extrapolar el significado a otros contextos.

Debido a lo anterior, lo que interesa conocer es ¿Qué significa el recurso de tirarse al suelo en el contexto de almuerzo? Para responder a esta pregunta la actividad del almuerzo es segmentada en tres partes: inicio, desarrollo y cierre de la actividad.

\subsection{Significado contextualizado del recurso tirarse al suelo}

\section{Durante inicio de actividad: "Sácame de este lugar", "No quiero comer"}

Durante el inicio de la actividad de almuerzo, es decir, al ingresar al comedor, puede utilizar dos veces el recurso de tirarse al suelo con dos significados distintos. Al entrar al comedor y tirarse al suelo el estudiante demanda servicios, significado que podría ser traducido como "sácame de este lugar". Cuando el estudiante entra en el comedor expresa el rechazo a estar en tal lugar, lo cual puede tener diversas causas, algunas de ellas pueden ser la presencia de estudiantes de otros cursos, la disposición del espacio, los sonidos, entre otros. Estas causas son posibles de indagar y determinar a partir de la interpretación del recurso "tirarse al suelo" en este contexto.

Al inicio de la actividad, acercarse a la mesa también ofrece información, significado interpretado como "No quiero comer". La diferenciación entre un significado y otro dependerá del contexto en que se ejecute, en este caso, cuando la comida del estudiante está servida en la mesa, el significado pasa de ser una demanda de servicios a una oferta de información "no quiero comer". 


\section{Durante el desarrollo de la actividad: "Déjame solo"}

Durante el desarrollo de la actividad el estudiante intenta escapar del comedor constantemente y utiliza el recurso "tirarse al suelo" cuando se lo impide el profesor o alguna asistente que se encuentre en el comedor. A la utilización de este recurso se le ha llamado "Déjame solo". En este contexto el estudiante está intentando buscar espacios para la auto regulación. Es altamente probable, considerando sus características de hipersensibilidad (Frith, 1994) que el exceso de estímulos sonoros y visuales en el contexto comedor generen una sobre estimulación lo que hace que el estudiante busque regularse al utilizar el recurso "tirarse al suelo" demandando la acción de dejarlo solo.

\section{Durante el final de la actividad: “¿Qué haremos?”, “QQuién me viene a buscar?”}

Durante el final de la actividad, ocurre la situación que es la más importante con relación a la mejora de la Calidad de Vida del estudiante, esto es utilizar el recurso "tirarse al suelo" después del término del periodo de almuerzo. Cuando el estudiante utiliza este recurso al final de la actividad de almuerzo se encuentra sólo o en compañía del profesor, lo cual implica que la actividad de almuerzo ya ha finalizado y el estudiante se encuentra solo. Esto significa que no utiliza el recurso como "déjame solo", tampoco lo utiliza como "no quiero comer" porque ya no tiene que comer, el plato está retirado de la mesa. Tampoco significa "sácame de aqui" puesto que el estudiante conoce la rutina de la actividad, es decir, sabe que es momento de salir.

En esta situación concreta, utilizando el recurso de "tirarse al suelo" el estudiante demanda información respecto a las actividades a realizar en el futuro inmediato, es decir, el momento en que los padres lo recogen del colegio. La información solicitada la interpretamos como"¿Quién me viene a buscar?" “¿Qué haremos?" Este significado es sumamente importante con relación a las descompensaciones emocionales y recursos comunicativos peculiares que presenta el estudiante durante el último periodo de clases, puesto que el joven expresa una relación afectiva/comunicativa diferente dependiendo de cuál de los dos padres lo retira de la escuela.

Esto se relaciona con las actividades que se realizarán posteriormente al periodo escolar ya que depende de cuál de los dos progenitores lo retire y de la actividad a realizarse de inmediato con él. Por ejemplo, si lo viene a buscar la mamá hay mayor probabilidad que su desplazamiento sea a la casa de forma inmediata, en donde la madre lo dejará en 
cuidado de otro familiar. Por otro lado, cuando lo retira el padre, suelen desplazarse hacia otros lugares antes de irse a la casa, como ir a supermercado o tiendas de comida rápida.

Esto implica que si se le otorgan los apoyos necesarios para anticipar de mejor manera la situación de salida de clase respecto a quién lo viene a buscar (papá o mamá) y qué actividad realizarán (ir al supermercado, a la casa, a comer, entre otros) es altamente probable que disminuya la ansiedad que presenta en esta situación y con esto disminuyan también sus descompensaciones emocionales durante el último periodo de clases. Así, al interpretar adecuadamente la necesidad que demanda el joven a través de este recurso que se presenta como disruptivo para su contexto escolar inmediato, se espera que este sea utilizado con menor o ninguna frecuencia al estar ya satisfecha su necesidad.

Como cierre de la interpretación del recurso "tirarse al suelo" es posible expresar que el recurso de "tirarse al suelo" lo utiliza principalmente como demanda de acciones durante el periodo de almuerzo en el comedor, y demanda de información durante el último periodo de la actividad de almuerzo, siendo esta última interpretación las más valiosa en tanto elemento para la mejora de la Calidad de Vida del estudiante. A continuación se presentan las principales conclusiones de esta investigación.

\section{Conclusiones}

Teniendo en cuenta la caracterización de las interacciones comunicativas de las personas con dentro del Espectro Autista desde la Semiótica Social y el paradigma Inclusivo, se logra develar el repertorio comunicativo de la persona de este estudio para contextos específicos en los que se desenvuelve. Esta descripción posibilita una mejor interpretación de las interacciones del estudio de caso, al conocer cuáles recursos utiliza en determinados contextos. Dicho conocimiento debería repercutir, por una parte, en una disminución de los malos entendidos por parte de los interlocutores quienes podrían ser más asertivos en el intercambio de significados $\mathrm{y}$, por otra, podría aminorar la frecuencia y duración de las situaciones en las que la persona dentro del Espectro Autista persevera en su conducta comunicativa sin obtener la respuesta buscada. La descripción del repertorio comunicativo o constelación (Quirós, 2006) de este estudiante en particular, va de la mano de la valoración de sus recursos para crear significados y de su potencial para comunicarse con los demás.

De esta manera, destacamos que la indagación en los recursos comunicativos de manera situada potencialmente podría repercutir en la mejora de la Calidad de Vida de la población estudiantil dentro del Espectro Autista, puesto que ayuda a la interpretación de sus 
necesidades, deseos y opiniones, respetando sus propios recursos comunicativos. En el caso de este estudio, resulta fundamental la interpretación del recurso "tirarse al suelo" como medio para la demanda de información, lo que otorga en primer lugar una visión explicativa de la razón por la cual genera esta conducta el estudiante durante el último periodo de clases, junto con una posibilidad práctica de generar un plan de apoyo individual que apunte a trabajar esta situación particular, así como otras, ya que se conoce los recursos y la intención comunicativa de estos recursos en ciertos contextos.

A modo general, cabe mencionar la necesidad de comprender que la visión que se tiene de las personas del espectro autista genera un impacto directo en las relaciones comunicativas que se tienen con ellas. Esto quiere decir, que es menester de la cultura en donde se desempeñan todas las personas que participan en la sociedad el educar en la valoración a la diversidad, teniendo en cuenta las distintas formas de ser en el mundo y, en el caso particular de este artículo, referido a la diversidad comunicativa, valorando positivamente las distintas formas y recursos que pueden ser utilizados en la comunicación.

En definitiva, se sostiene que la manera más adecuada, responsable y ética respecto a cómo investigar las interacciones de las personas dentro del Espectro Autista con el fin de mejorar su Calidad de Vida corresponde al Análisis Multimodal del Discurso desde la Semiótica Social. Ya que permite interpretar todos los recursos que posee una persona para significar y comunicarse en contextos de situación determinados, respetando así las particularidades personales y sociales de cada quien.

Los hallazgos presentados pueden aportar a múltiples ejes de acción: intervención y apoyo especializado, funciones psicológicas, detección y diagnóstico, apoyos educativos, cambios en la cultura escolar y familiar entre otros. Pero lo más importante consiste en un cambio de mirada respecto a qué significa comunicar y en qué medida esta noción de comunicación afecta a nuestra forma de concebir lo humano y, por consiguiente, afecta la forma de relacionarnos con las personas que presentan el espectro autista.

\section{Referencias}

Bates, Elizabeth, Benigni, Laura, Betherton, Inger, Camaioni, Luigia y Volterra, Virginia. (1979). The emergence of symbols: Cognition and comunication in infancy. New York: Academic Press.

Belinchón, Mercedes. (2010). Investigaciones sobre Autismo en Español: problemas y perspectivas. Madrid: Universidad Autónoma de Madrid. 
Booth, Tony y Ainscow, Mel. (2002). Índice de Inclusión, desarrollando el aprendizaje y la participación en las escuelas. Bristol: CSIE.

Cabrera, Ana Lilia. (2012). Paradigmas de la Educación Especial. Tlalnepantla, México: Red Tercer Milenio.

Dreyfus, Shoshana. (2006). When there is no speech: a case study of the nonverbal multimodal comunication of a child with an intellectual disability. Wollongong: University of Wollongong.

Dreyfus, Shoshana. (2011). When there is no speech: nonverbal comunication in intellectual disability. Londres: Equinox.

Echeverría, Eva, González, Daniela, Guajardo, Viviana, Martínez, Marcela y Rodríguez, Pamela. (2011). ¿Qué deben leer y escribir los estudiantes de cuarto Básico para aprender en Comprensión del medio social y cultural? Descripción Multimodal del material de trabajo y estrategias de Mediación. Valparaíso: PUCV.

Eggins, Suzanne. (2004). Introduction to systemic functional linguistics (2da ed.). Londres: Continuum

Eggins, Suzanne y Martin, Jim. (2003). El contexto como género: una perspectiva lingüística funcional. Revista Signos, 36(54), 185-205.

Frith, Uta. (1994). Autismo hacia una explicación del enigma. Madrid: Alianza.

Gutiérrez, Rosa María. (2011). Descripción sistémico-funcional y gramática multiregistro. Estudios filosóficos 47, 59-82

Halle, Jim, Brady, Nancy y Drasgow, Erick. (2004). Enhancing socially adaptive communicative repairs of beginning communicators with disabilities. America Journal of Speech-Language Pathology, 13, 43-54.

Halliday, Michael. (1978). Language as a Social Semiotic. The Social Interpretation of Language and Meaning. London: Edward Arnold.

Halliday, Michael \& Matthiessen Christian. (1994). An Introduction to Sistemic Functional Linguistics. Londres: Continuum.

Halliday, Michael. (1994). Introduction to Sistemic Functional Linguistics. Londres: Continuum.

Hodge, Robert y Kress, Gunther. (1988). Social Semiotics. Cambridge: Polity.

Kress, Gunther y Van Leuween, Theo. (1996). The grammar of Visual Design. Londres: Routledge.

Kress, Gunther, y Van Leween, Theo. (2001). Multimodal DiscourseThe modes and the media contemporary comunications. Londres: Arnold. 
Kress, Gunther. (2010). Multimodality: A social semiotic approach to contemporary communication. London: Routledge.

Martos, Juan y Riviere, Ángel. (2001). Prólogo. En Juan Martos y Ángel Riviere, (eds.), Autismo: Comprensión y explicación actual (pp. 13-16). Madrid: Instituto de Migraciones y servicios sociales

Mella, Orlando. (1998). Naturaleza y orientaciones teórico-metodológicas de la Investigación Cualitativa. Recuperado de http://www.reduc.cl/wpcontent/uploads/2014/08/NATURALEZA-Y-ORIENTACIONES.pdf

Moss, Gillian. (2010). Un modelo funcional del lenguaje. En Diana Chamorro y Norma Barletta (eds.), Texto y aprendizaje: enredos y desenredos (pp. 15- 26). Barranquilla: Editorial Universidad del Norte.

Oteiza, Teresa. (2006). El discurso pedagógico de la historia: un análisis lingüístico sobre la construcción ideológica de la historia de Chile (1980-2001). Chile: Frasis.

Quirós, Amaryllis. (2006). Repertorios comunicativos en la constelación autista. Actualidades en Psicología, 20, 90-104.

Riviere, Ángel y Núñez, María. (1996). La mirada mental. Argentina: Aique.

Riviere, Angel. (1990). Origen y desarrollo de la función simbólica en el niño. En Jesús Palacios, Álvaro Marchiesi y César Coll (comps.), Desarrollo Psicológico y educación (I Psicología Evolutiva (pp. 329-360). Madrid: Alianza.

Riviere, Angel. (2000). El niño pequeño con Autismo. Madrid: APNA.

Schalock, Robert. (2011). Discapacidad Intelectual: definición, clasificación y sistemas de apoyo. Madrid: Alianza

Tamarit, Javier. (2005). Autismo: modelos educativos para una vida de calidad. Rev neurol, $40(1), 181-186$.

Vigotsky, Lev. (1998). Lenguaje y pensamiento. La Habana: Pueblo y educación.

Wing, Lorna. (1997). The History of ideas of Autism. Autism, 13-23. 